\title{
Central Blood Pressure and Chronic Kidney Disease Progression
}

\author{
Debbie L. Cohen and Raymond R. Townsend \\ Renal, Electrolyte and Hypertension Division, University of Pennsylvania, 1 Founders Building, 3400 Spruce Street, \\ Philadelphia, PA 19104, USA \\ Correspondence should be addressed to Debbie L. Cohen, debbie.cohen@uphs.upenn.edu
}

Received 8 December 2010; Accepted 5 January 2011

Academic Editor: Hiromichi Suzuki

Copyright ( $) 2011$ D. L. Cohen and R. R. Townsend. This is an open access article distributed under the Creative Commons Attribution License, which permits unrestricted use, distribution, and reproduction in any medium, provided the original work is properly cited.

\begin{abstract}
Hypertension, diabetes, and proteinuria are well-recognized risk factors for progressive kidney function loss. However, despite excellent antihypertensive and antidiabetic drug therapies, which also often lower urinary protein excretion, there remains a significant reservoir of patients with chronic kidney disease who are at high risk for progression to end-stage kidney disease. This has led to the search for less traditional cardiovascular risk factors that will help stratify patients at risk for more rapid kidney disease progression. Among these are noninvasive estimates of vascular structure and function. Arterial stiffness, manifested by the pulse wave velocity in the aorta, has been established in a number of studies as a significant risk factor for kidney disease progression and cardiovascular endpoints. Much less well studied in chronic kidney disease are measures of central arterial pressures. In this paper we cover the physiology behind the generation of the central pulse wave contour and the studies available using these approaches and conclude with some speculations on the rationale for why measurements of central pressure may be informative for the study of chronic kidney disease progression.
\end{abstract}

\section{Introduction}

The presence of chronic kidney disease (CKD) enhances the likelihood of cardiovascular disease (CVD) hospitalizations and death [1]. Even modest kidney function impairment is associated with a significant increase in cardiovascular risk [2]. The relationship of traditional factors for CVD such hypertension, diabetes, and proteinuria also contribute, to progressive renal function decline. However the significantly higher risk of CVD in patients with CKD is not fully explained by traditional factors; thus, there remains interest in investigating the association of less traditional risk factors, such as central blood pressure measurements, with both CVD and the progression of CKD [3].

The last three decades have witnessed a remarkable increase in the usage of invasive and noninvasive methods to estimate central blood pressure profiles [6]. Many of these approaches are based on tonometric assessment of waveforms or the velocity of pulse wave travel in the aorta. A recent meta-analysis of the usefulness of central pressure measurements for predicting CVD events concluded that the augmentation index provided independent CVD predictive utility when modeled together with traditional blood pressure measures for this outcome [7]. These devices have facilitated the incorporation of measures of central blood pressure into a number of observational prospective studies in the CKD population. Several studies in the end-stage kidney disease (ESKD) population have testified to the value of central pressure measurements for CVD events but less research has focused on the predialysis CKD population, particularly with CKD progression as the outcome of interest. This paper will focus on cross-sectional and longitudinal associations between central blood pressure measurements in the predialysis CKD population and specifically directed toward CKD outcomes. Several approaches have been taken to study arterial structure and function. These are generally categorized as:

(1) those that measure large artery function as reflected by determinations of large artery stiffness which is usually assumed to be represented by the velocity of pulse wave travel in the vessel(s) of interest [8]

(2) those that measure the effects of aspects of wave reflection such as augmentation index, central systolic and central pulse pressure [8]. 


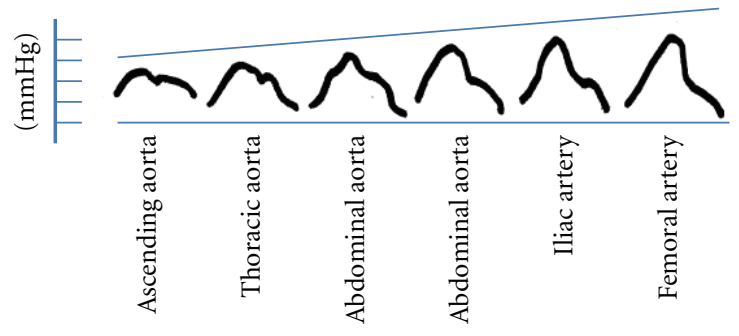

FIGURE 1: Changes in arterial pressure wave contour between ascending aorta and femoral artery (adapted from reference [4]).

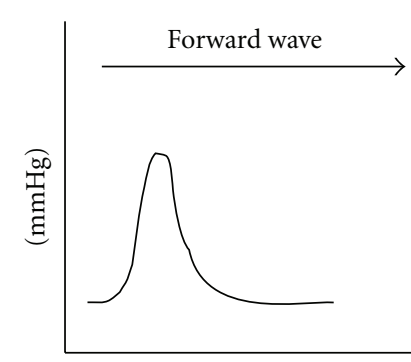

Time (ms)
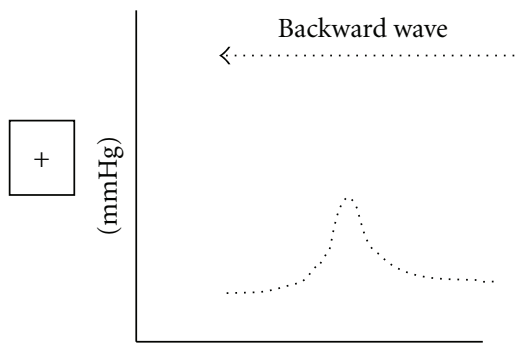

Time (ms)

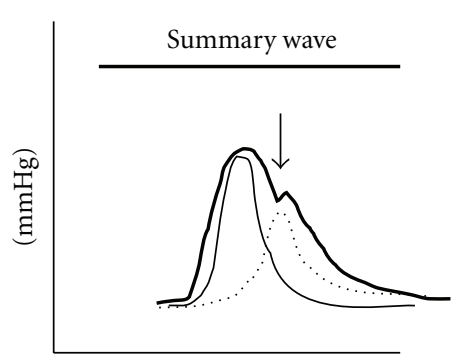

Time (ms)

FIGURE 2: Pictorial representation of summation (right) effects on the arterial pressure waveform resulting from forward (left) and backward (i.e., "reflected"; middle) wave integration.

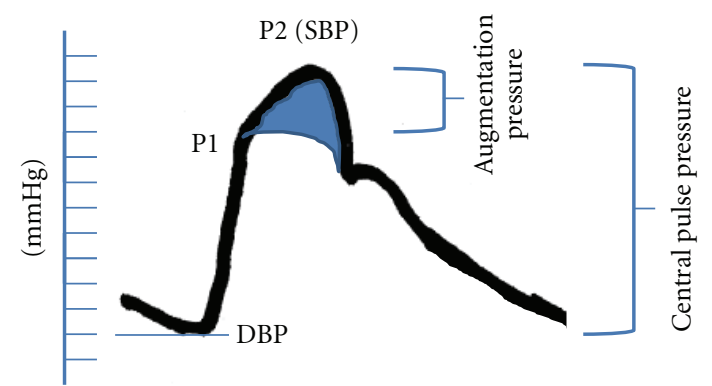

FIGURE 3: Pictorial representation of central vessel waveform. P1 represents the pressure effected by the forward traveling wave. P2 minus $\mathrm{P} 1$ represents the amount of systolic pressure augmentation attributed to the reflected wave. P2 minus the diastolic blood pressure (DBP) is the central pulse pressure. The augmentation pressure (P2-P1) divided by the central pulse pressure is the Augmentation Index. The shaded area in the waveform estimates the effect of the reflected wave form on systolic workload.

This paper will focus mainly on investigations using the second approach. In some studies both have been used, but these tend to be exceptions rather than the rule.

\section{Wave Reflection}

Before reviewing studies using technology that quantify the effect of wave reflection on the central circulation it is important to review, briefly, the physiology of wave reflection and define the terms used in this area of research.

The shape, and the magnitude of the arterial waveform changes as the wave travels from the heart to the periphery as shown in Figure 1. For the purpose of the current paper note that the pulse pressure (defined as the systolic pressure-diastolic pressure) increases as the pressure wave travels to the periphery. This phenomenon is termed pulse pressure augmentation and results from the interaction of the forward and backward traveling pressure waves which, summate algebraically as shown in Figure 2. Depending on where the measurement is made in the circulation the waveform will vary in shape and magnitude depending on its relative location to wave reflecting sites. Wave reflection results from changes in vascular impedance (defined as the relationship of pressure divided by flow). Things that change vascular impedance include branching, plaque, and changes in vascular caliber for example. If there were no changes in vascular impedance, each pressure wave would simply assume the shape of a flow wave. Since there are changes in vascular impedance, their effect modifies the forward traveling pressure wave as shown in Figure 3. The change in systolic contour in the shaded area of Figure 3 represents the effect of wave reflection. This is usually quantified either by determining the difference between the peak systolic pressure (P2) divided by the central pulse pressure (called the augmentation index, $(A I x))$ or by determining the degree of augmented pressure by subtracting P1 from P2 (called the augmentation pressure, $(A P))$. The rest of Figure 3 depicts the terminology used for a representative central pressure waveform. Figure 4 shows typical waveforms obtained from the radial artery with corresponding aortic pressure profiles illustrating the effects of age on waveform reflection (in a CKD cohort). Other determinants of wave reflection include heart rate [9], height/gender [4, 10], medications [11], and various comorbidities such as hypertension, diabetes, and other standard CVD risk factors [12]. Several reviews are available to consult for a more in depth study of reflected wave physiology [13-15]. 

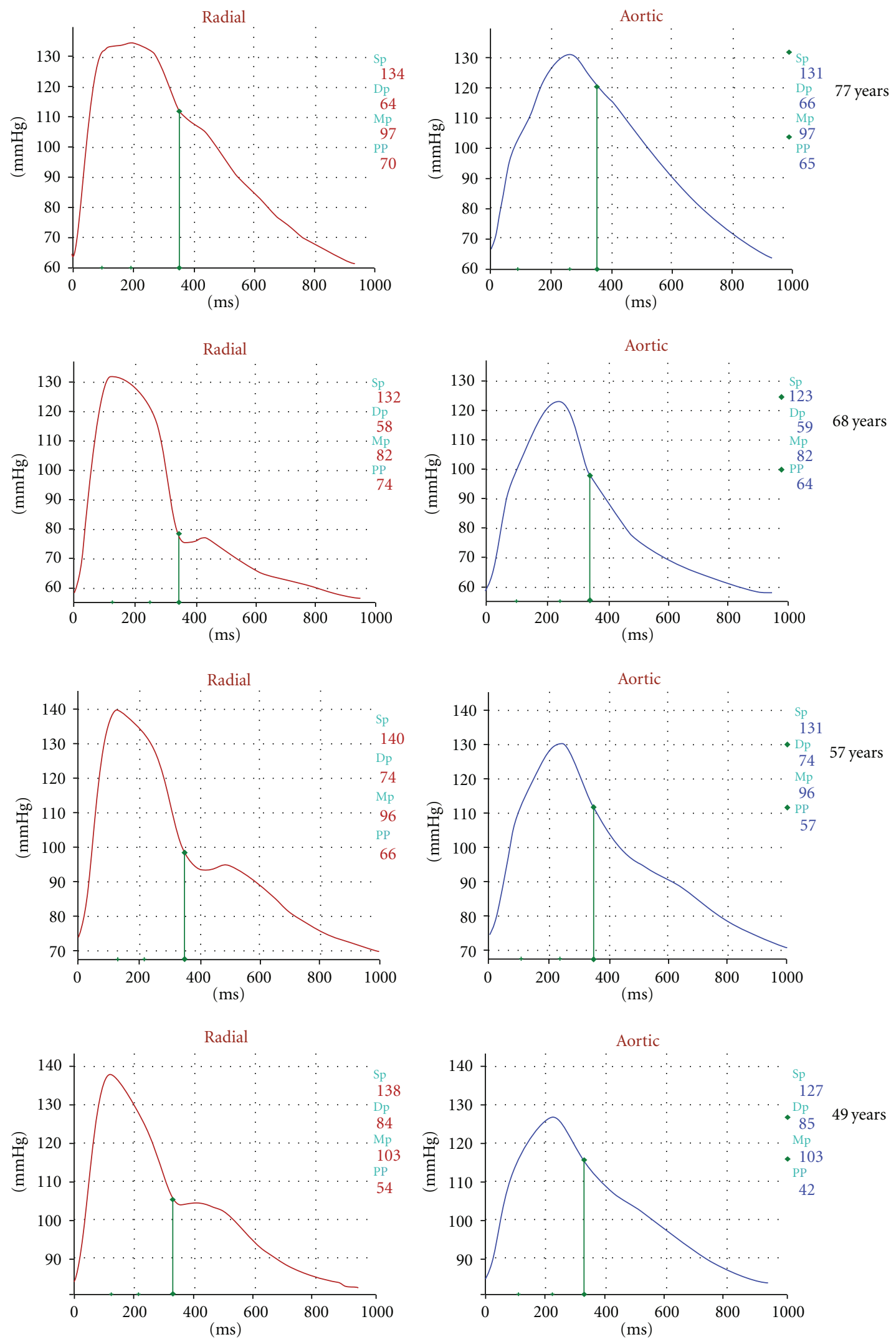

FIgure 4: Continued. 

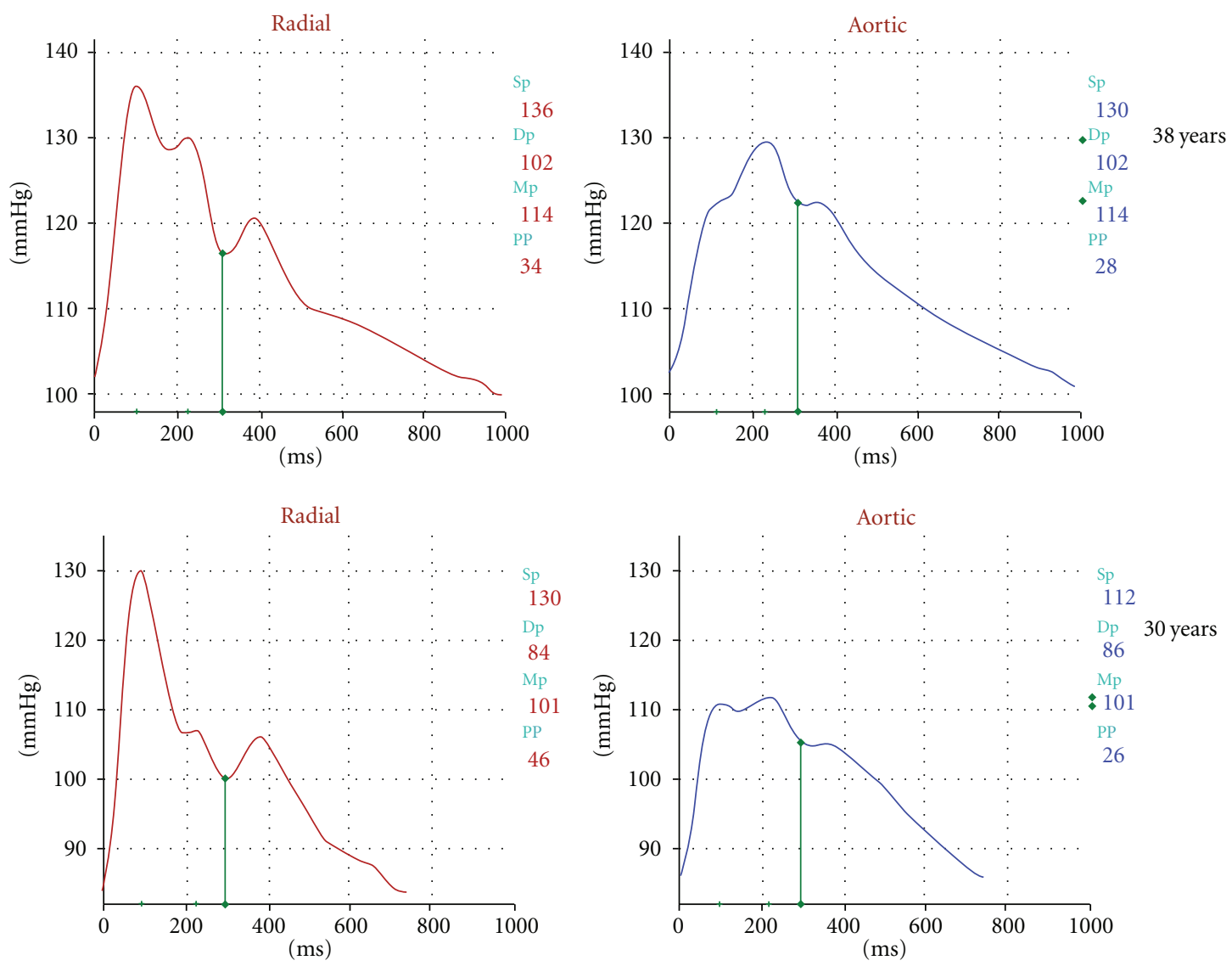

FIGURE 4: Representative wave forms showing the effect of aging on the radial (left) and aortic (right) pulse contours from subjects studied in the CRIC.

\section{Studies of Central Pressure: Profiling CKD Progression}

One of the earliest investigations of arterial waveform analysis and CKD progression was undertaken by Takenaka et al. in a Japanese cohort of nondiabetic CKD patients using radial artery tonometry [16]. In 41 patients with a mean baseline creatinine clearance of $52 \mathrm{~mL} / \mathrm{min}$ they reported that a higher radial AIx value, along with higher baseline urine protein excretion were the only significant factors in their multiple regression analysis for the decline in creatinine clearance 1 year later.

Taal et al. building on the Takenaka study, expanded the relevant variables studied in a slightly smaller sample size ( $n=35$ ) of stage 4 and 5 CKD patients and pursued the endpoint of ESKD defined as commencing dialysis more than 30 days after enrollment [17]. Over a period of slightly more than one year 22 of the 35 study participants commenced dialysis. Baseline eGFR, urine protein excretion, cigarette usage, higher PWV and an AIx above the median value (of $43 \%$ ) were all shown to be independent predictors for progression onto dialysis.

The chronic renal insufficiency cohort (CRIC) ancillary study has measured central aortic PP (CPP) using radial tonometry since 2005. One of the aims of the study was to determine clinical factors independently associated with CPP in CKD and to evaluate how well brachial PP correlates with CPP in a large ethnically diverse population of CKD. The CRIC study measured central pulse pressure crosssectionally in 2531 participants in the CRIC study to determine correlates of the magnitude of central pulse pressure in the setting of CKD. In the cross-sectional analysis central pulse pressure measurements tertiles were $<36 \mathrm{mmHg}, 36-$ $51 \mathrm{mmHg}$ and $>51 \mathrm{mmHg}$ with an overall mean CPP of $46 \mathrm{mmHg}$ and a mean brachial PP of $57 \mathrm{mmHg}$. An increasing proportion of those subjects within each worsening CKD stage had a central pulse pressure $\geq 50 \mathrm{mmHg}$ (Figure 5) [5].

Data from the CRIC study were analyzed longitudinally to determine which central hemodynamic measure (central systolic pressure, AIx, AP, and central pulse pressure) had the best predictive potential for death and kidney function decrement endpoints. To date the following data have only been presented in abstract form [18].

Approximately half of the CRIC participants were females with a mean age of 60 years and a mean eGFR of $41 \mathrm{~mL} / \mathrm{min} / 1.73 \mathrm{~m}^{2}$. The events analyzed were (1) death, (2) ESKD $(n=99)$ or (3) a $50 \%$ reduction in eGFR from baseline or ESKD ("RENAL1" endpoint; $n=129$ ). Death was treated as a censoring event for the renal events. Figure 6 (redrawn from the abstract [18]) displays the hazard ratios 


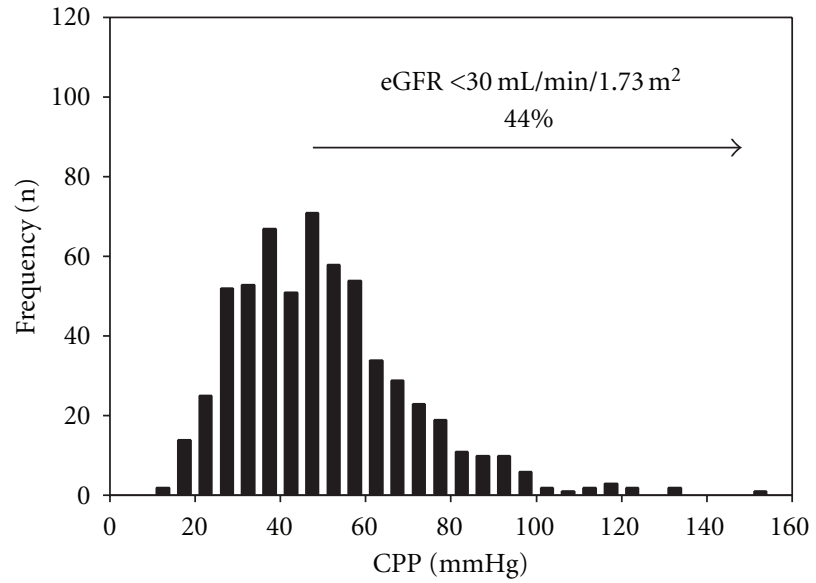

(a)

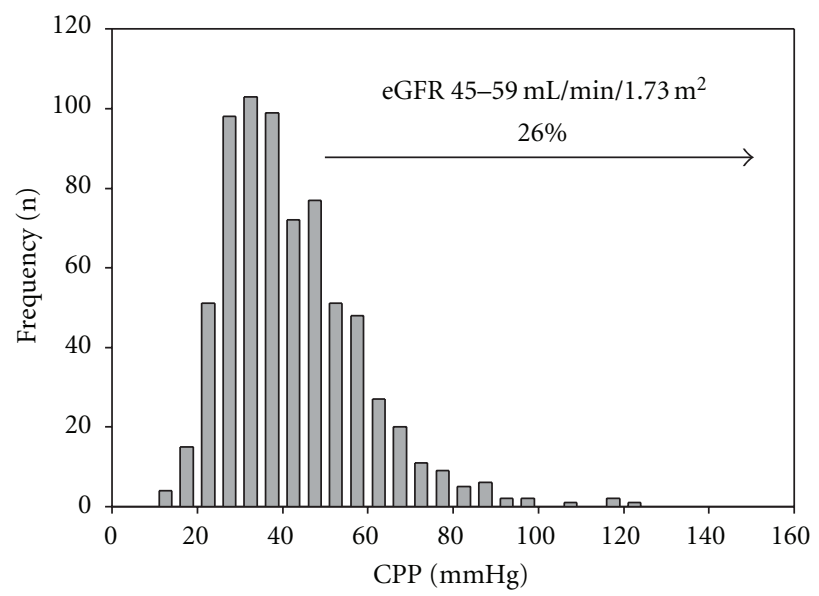

(c)

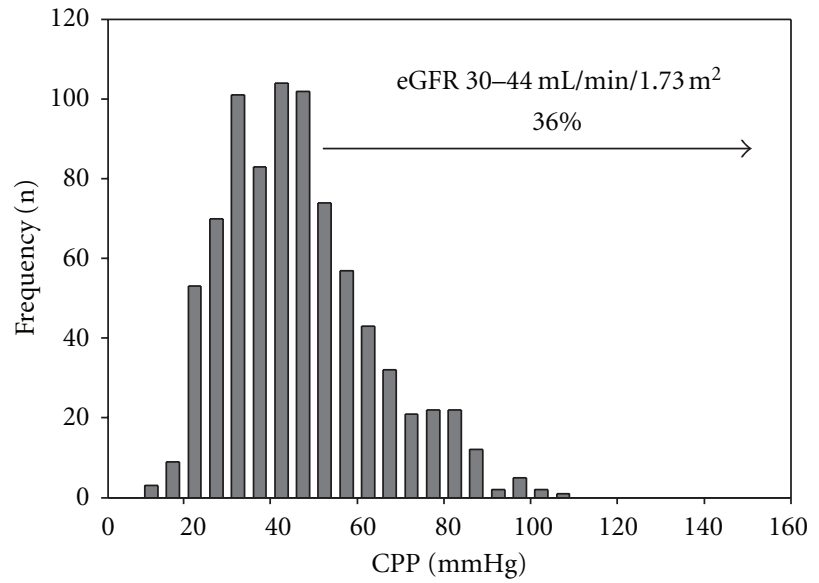

(b)

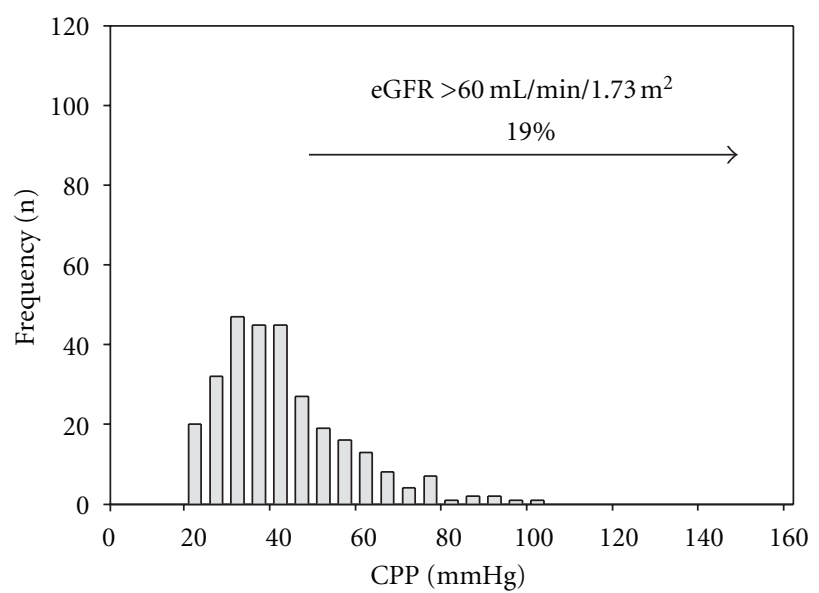

(d)

FIGURE 5: Increasing prevalence of central pulse pressure values $>50 \mathrm{mmHg}$ among 2531 participants in the Chronic Renal Insufficiency Cohort (CRIC) (redrawn from [5]).

(adjusted for age, race, sex, diabetes, eGFR, proteinuria, and clinical site) with the different independent variables categorized using common clinical cut-points for brachial systolic/pulse pressures, and central aortic systolic/pulse/ augmentation pressures and aortic pulse wave velocity. These findings suggested that in CKD

(1) increasing PWV predicts ESKD,

(2) central pressures also predict ESKD,

(3) central pulse pressure appears to predict ESKD best among the four selected measures of central pressure.

\section{Speculations}

Given the paucity of studies of CKD progression, and the limitations of noninvasive technology to pursue mechanisms of progressive kidney damage, it is difficult to state with certainty how increasing pulse pressure mediates increasing kidney function loss. Loutzenhiser et al. and others, have stressed relative importance of afferent arterial myogenic tone, and its relationship to tubuloglomerular feedback [19]. It stands to reason then, that in an organ like the kidney, whose blood flow (relative to mass) has been described as "torrential" [20], increasing pulse pressure would act like a progressively heavy hammer slamming into the delicate capillary network of the glomerulus particularly when the afferent arteriole fails as a gatekeeper to buffer the incoming pressure waveform. Their work in animal models suggest the systolic pressure is important in influencing afferent arteriolar myogenic responses [21]. Whether this is the most important hemodynamic parameter in humans, especially given the variety of processes associated with CKD (diabetes, glomerulonephritis, etc.), is not as clear. The morphometric work of Gary Hill supports this concept. He has shown that larger afferent arterioles (on kidney biopsy) in hypertension correlate positively to the size of the glomerulus and also predict the likelihood of finding focal sclerosis [22]. Moreover, it seems plausible to these authors that when a higher pulse pressure is delivered at a greater velocity (courtesy of 


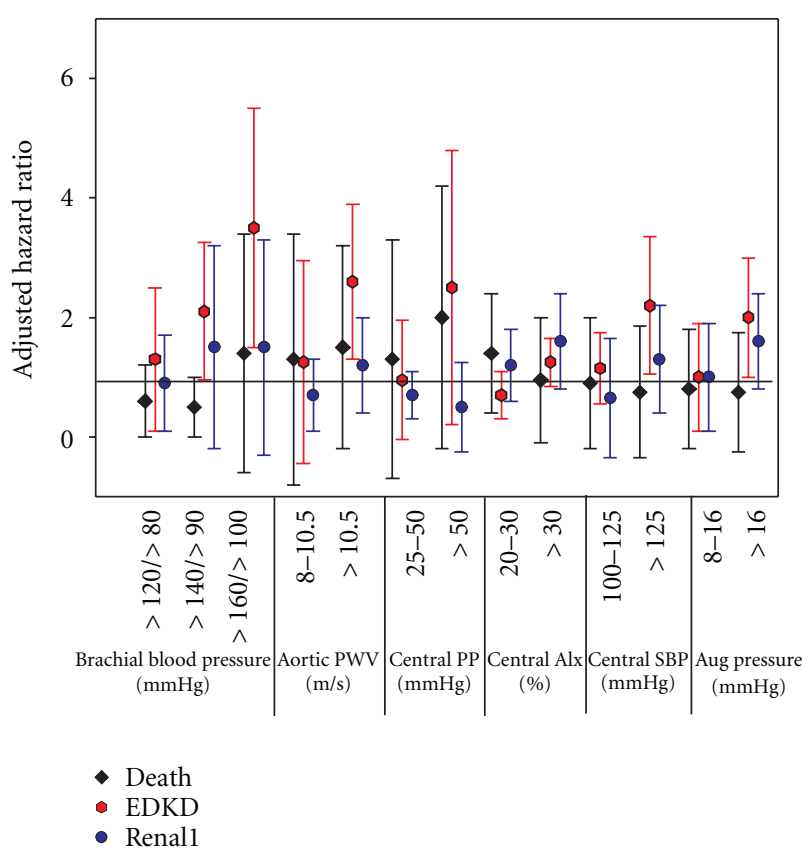

FIGURE 6: Referent groups are $<120 /<80$ for brachial BP, $<8 \mathrm{~m} / \mathrm{sec}$ for aortic pulse wave velocity (PWV), $<25 \mathrm{mmHg}$ for central pulse pressure (PP), $<20 \%$ for central augmentation index (AIx), $<100$ for central systolic blood pressure (SBP), and $<8 \mathrm{mmHg}$ for the augmented (Aug) central pressure wave. Endpoints were death (black diamond), end-stage kidney disease (ESKD, red hexagon) and doubling of serum creatinine or ESKD or death (blue circle).

increasing central arterial stiffness) the damaging effect of the higher pulse pressure would be amplified by the higher velocity of delivery because of the greater energy dispersion into tissues associated with higher PWV values.

\section{References}

[1] A. S. Go, G. M. Chertow, D. Fan, C. E. McCulloch, and C. Y. Hsu, "Chronic kidney disease and the risks of death, cardiovascular events, and hospitalization," New England Journal of Medicine, vol. 351, no. 13, pp. 1296-1305, 2004.

[2] M. G. Shlipak, R. Katz, M. J. Sarnak et al., "Cystatin C and prognosis for cardiovascular and kidney outcomes in elderly persons without chronic kidney disease," Annals of Internal Medicine, vol. 145, no. 4, pp. 237-246, 2006.

[3] R. R. Townsend and H. I. Feldman, "CKD: past problems, current challenges and future facets," NephSAP, vol. 8, no. 4, pp. 1-5, 2009.

[4] C. J. Mills, I. T. Gabe, J. H. Gault et al., "Pressure-flow relationships and vascular impedance in man," Cardiovascular Research, vol. 4, no. 4, pp. 405-417, 1970.

[5] R. R. Townsend, J. A. Chirinos, A. Parsa et al., "Central pulse pressure in chronic kidney disease: a chronic renal insufficiency cohort ancillary study," Hypertension, vol. 56, no. 3, pp. 518-524, 2010.

[6] G. M. London, A. P. Guerin, B. M. Pannier, S. J. Marchais, and F. Metivier, "Body height as a determinant of carotid pulse contour in humans," Journal of Hypertension, vol. 10, no. 6, pp. S93-S95, 1992.
[7] C. Vlachopoulos, K. Aznaouridis, M. F. O’Rourke, M. E. Safar, K. Baou, and C. Stefanadis, "Prediction of cardiovascular events and all-cause mortality with central haemodynamics: a systematic review and meta-analysis," European Heart Journal, vol. 31, no. 15, pp. 1819-1822, 2010.

[8] S. Laurent, J. Cockcroft, L. Van Bortel et al., "Expert consensus document on arterial stiffness: methodological issues and clinical applications," European Heart Journal, vol. 27, no. 21, pp. 2588-2605, 2006.

[9] I. B. Wilkinson, H. MacCallum, L. Flint, J. R. Cockcroft, D. E. Newby, and D. J. Webb, "The influence of heart rate on augmentation index and central arterial pressure in humans," Journal of Physiology, vol. 525, no. 1, pp. 263-270, 2000.

[10] S. J. Marchais, A. P. Guerin, B. M. Pannier, B. I. Levy, M. E. Safar, and G. M. London, "Wave reflections and cardiac hypertrophy in chronic uremia: influence of body size," Hypertension, vol. 22, no. 6, pp. 876-883, 1993.

[11] A. D. Protogerou, G. S. Stergiou, C. Vlachopoulos, J. Blacher, and A. Achimastos, "The effect of antihypertensive drugs on central blood pressure beyond peripheral blood pressure. Part II: evidence for specific class-effects of antihypertensive drugs on pressure amplification," Current Pharmaceutical Design, vol. 15, no. 3, pp. 272-289, 2009.

[12] C. M. McEniery, Yasmin, B. McDonnell et al., "Central pressure: variability and impact of cardiovascular risk factors the anglo-cardiff collaborative trial II," Hypertension, vol. 51, no. 6, pp. 1476-1482, 2008.

[13] M. F. O’Rourke, "From theory into practice: arterial haemodynamics in clinical hypertension," Journal of Hypertension, vol. 20, no. 10, pp. 1901-1915, 2002.

[14] R. R. Townsend, "Analyzing the radial pulse waveform: narrowing the gap between blood pressure and outcomes," Current Opinion in Nephrology and Hypertension, vol. 16, no. 3, pp. 261-266, 2007.

[15] W. F. NIchols and M. F. O'Rourke, "Wave reflections," in McDonald's Blood Flow in Arteries: Theoretical, Experimental and Clinical Principles, W. F. Nichols and M. F. O'Rourke, Eds., pp. 154-233, Oxford University Press, London, UK, 5th edition, 2005.

[16] T. Takenaka, T. Mimura, Y. Kanno, and H. Suzuki, "Qualification of arterial stiffness as a risk factor to the progression of chronic kidney diseases," American Journal of Nephrology, vol. 25, no. 5, pp. 417-424, 2005.

[17] M. W. Taal, M. K. Sigrist, A. Fakis, R. J. Fluck, and C. W. McIntyre, "Markers of arterial stiffness are risk factors for progression to end-stage renal disease among patients with chronic kidney disease stages 4 and 5," Nephron Clinical Practice, vol. 107, no. 4, pp. c177-c181, 2007.

[18] R. R. Townsend, J. A. Chirinos, S. M. Sozio et al., "Comparison of standard blood pressure, pulse wave velocity and central hemodynamics in CKD progression: a CRIC ancillary study," Hypertension, vol. 56, article e68, 2010.

[19] R. Loutzenhiser, K. Griffin, G. Williamson, and A. Bidani, "Renal autoregulation: new perspectives regarding the protective and regulatory roles of the underlying mechanisms," American Journal of Physiology, vol. 290, no. 5, pp. R1153R1167, 2006.

[20] M. F. O'Rourke and M. E. Safar, "Relationship between aortic stiffening and microvascular disease in brain and kidney: cause and logic of therapy," Hypertension, vol. 46, no. 1, pp. 200-204, 2005. 
[21] R. Loutzenhiser, A. Bidani, and L. Chilton, "Renal myogenic response: kinetic attributes and physiological role," Circulation Research, vol. 90, no. 12, pp. 1316-1324, 2002.

[22] G. S. Hill, D. Heudes, C. Jacquot, E. Gauthier, and J. Bariety, "Morphometric evidence for impairment of renal autoregulation in advanced essential hypertension," Kidney International, vol. 69, no. 5, pp. 823-831, 2006. 


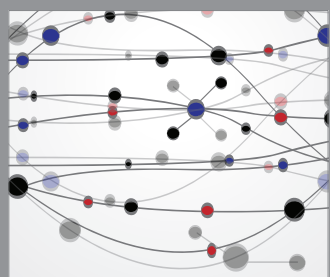

The Scientific World Journal
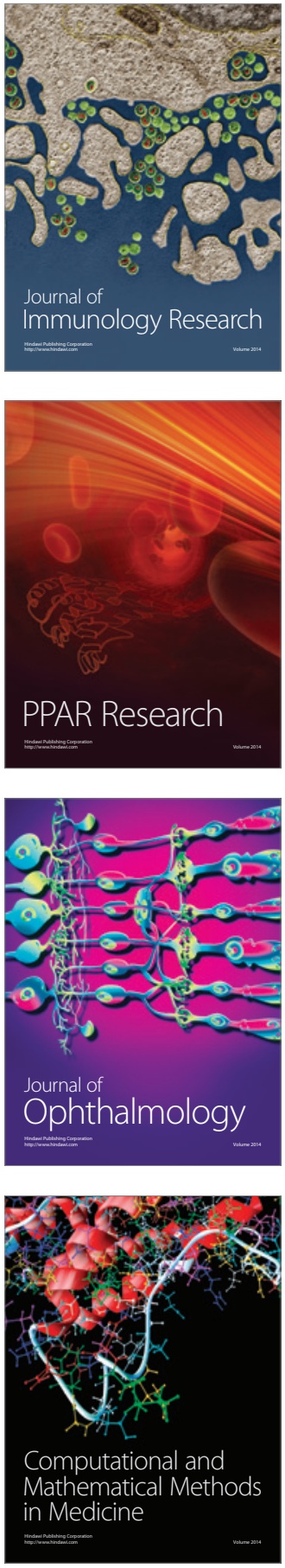

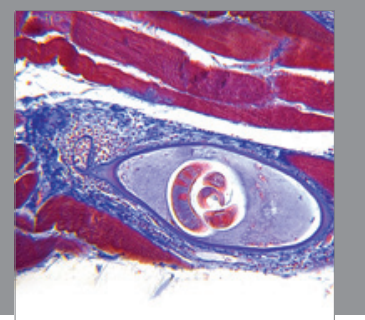

Gastroenterology

Research and Practice
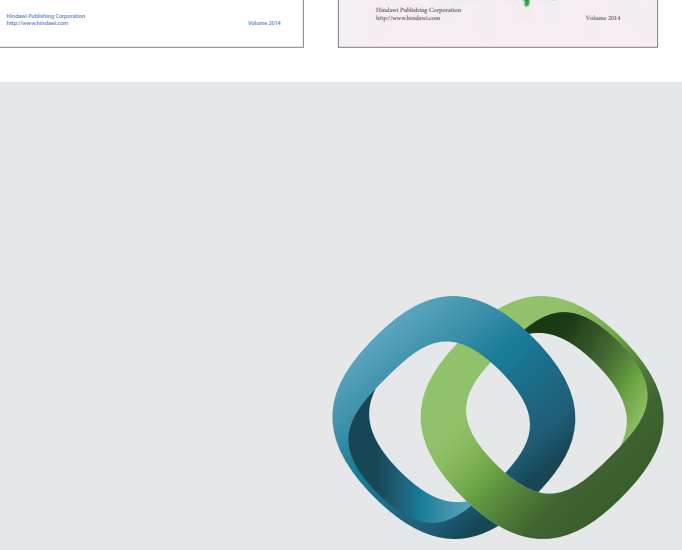

\section{Hindawi}

Submit your manuscripts at

http://www.hindawi.com
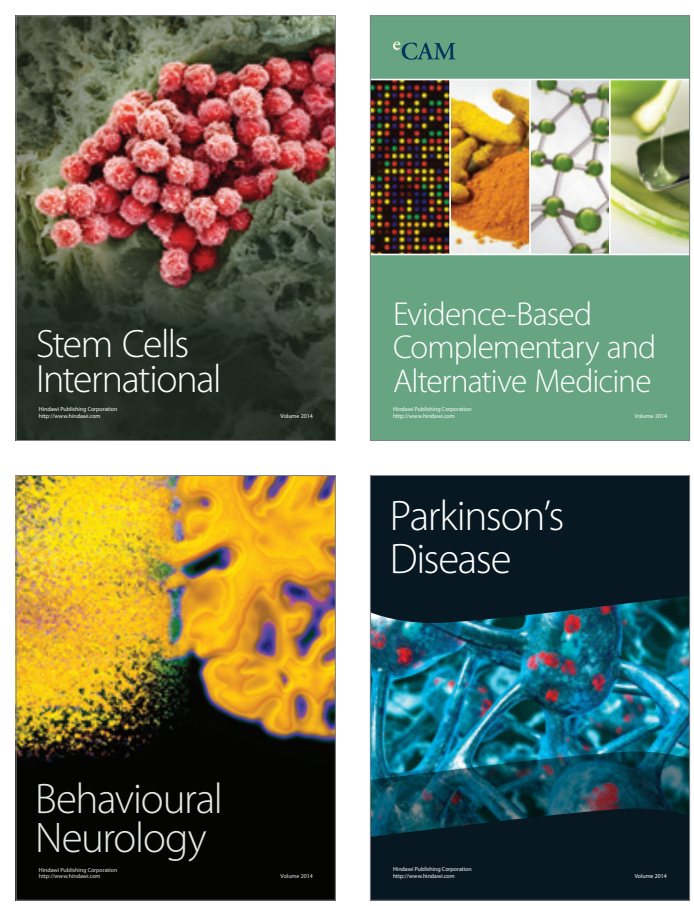

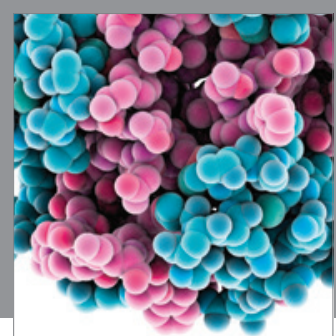

Journal of
Diabetes Research

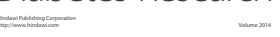

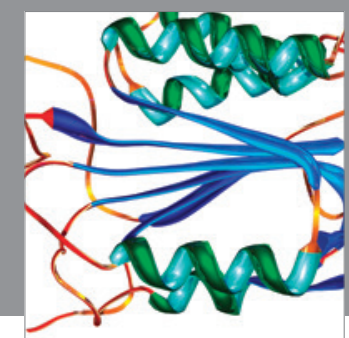

Disease Markers
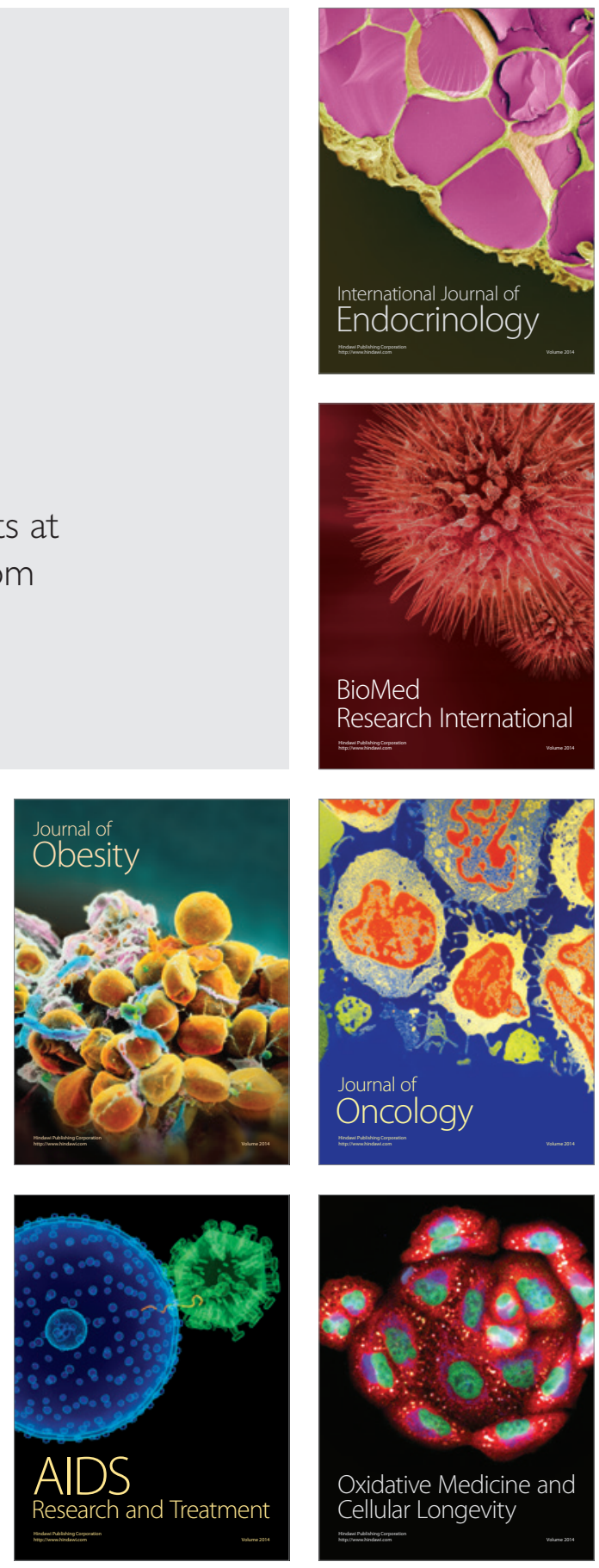